\title{
Handover patterns: an observational study of critical care physicians
}

\author{
Roy $\|_{a n}{ }^{*}$, Curtis D LeBaron ${ }^{2}$, Marlys K Christianson ${ }^{3}$, Daren K Heyland ${ }^{1}$, Andrew Day ${ }^{4}$ and Michael D Cohen ${ }^{5}$
}

\begin{abstract}
Background: Handover (or 'handoff') is the exchange of information between health professionals that accompanies the transfer of patient care. This process can result in adverse events. Handover 'best practices', with emphasis on standardization, have been widely promoted. However, these recommendations are based mostly on expert opinion and research on medical trainees. By examining handover communication of experienced physicians, we aim to inform future research, education and quality improvement. Thus, our objective is to describe handover communication patterns used by attending critical care physicians in an academic centre and to compare them with currently popular, standardized schemes for handover communication.
\end{abstract}

Methods: Prospective, observational study using video recording in an academic intensive care unit in Ontario, Canada. Forty individual patient handovers were randomly selected out of 10 end-of-week handover sessions of attending physicians. Two coders independently reviewed handover transcripts documenting elements of three communication schemes: SBAR (Situation, Background, Assessment, Recommendations); SOAP (Subjective, Objective, Assessment, Plan); and a standard medical admission notē. Frequency and extent of questions asked by incoming physicians wêre measured as well. Analysis consisted of descriptive statistics.

Results: Mean ( \pm standard deviation) duration of patient-specific handovers was 2 min $58 \mathrm{sec}( \pm 57 \mathrm{sec}$ ). The majority of handovers' content consisted of recent and current patient status. The remainder included physicians' interpretations and advice. Questions posed by the incoming physicians accounted for 5.8\% ( $\pm 3.9 \%)$ of the handovers' content. Elements of all three standardized communication schemes appeared repeatedly throughout the handover dialogs with no consistent pattern. For example, blocks of SOAP's Assessment appeared 5.2 ( \pm 3.0) times in patient handovers; they followed Objective blocks in only $45.9 \%$ of the opportunities and preceded Plan in just $21.8 \%$. Certain communication elements were occasionally absent. For example, SBAR's Recommendation and admission note information about the patient's Past Medical History were absent from 22 (55.0\%) and 20 (50.0\%), respectively, of patient handovers.

Conclusions: Clinical handover practice of faculty-level critical care physicians did not conform to any of the three predefined structuring schemes. Further research is needed to examine whether alternative approaches to handover communication can be identified and to identify features of high-quality handover communication.

Keywords: Adverse effects, Communication, Safety, Standardization, Video Recording

\section{Background}

Handover, or an equivalent term 'handoff', is the exchange between health professionals of information about a patient accompanying either a transfer of control over, or of responsibility for, the patient [1]. Miscommunication during transfer of care for hospitalized

\footnotetext{
* Correspondence: ilanr@kgh.kari.net

'Department of Medicine and Critical Care Program, Queen's University, Kingston General Hospital, Etherington Hall, Room 1005, 94 Stuart Street, Kingston, ON, Canada, K7L 3N6

Full list of author information is available at the end of the article
}

patients is common and can result in adverse events [2-7].

Guidelines and recommendations for handover practices have been proposed by patient safety organizations around the world [8-13]. In particular, the use of standardized approaches during handovers, including mnemonics that establish topics and their sequence, has been promoted and adopted by accreditation committees $[14,15]$.

\section{Biomed Central}


However, most suggested recommendations are based on expert opinions and have not been shown to improve communication effectiveness or patient safety. Furthermore, the available knowledge on physicians' handovers is based predominantly on studies of medical trainees $[11,16,17]$. In a recent literature review, Reisenberg and colleagues identified only one study that did not involve residents [17]. Our study seeks to uncover the existing practices of experienced physicians (attending physicians rather than residents) with an aim to compare their communication approaches to proposed standards. Our research acknowledges the inherent wisdom of everyday practice - that is, we recognize that people and professionals have an abundant capacity to figure out what works for them in a particular situation [18-20]. The intensive care unit (ICU), replete with potent and complex interventions, is a good place to study naturally occurring handovers. Accordingly, we conducted an observational study to characterize and assess handover patterns among attending critical care physicians. In the present study we consider a specific issue of direct policy significance: Since many calls have been issued for standardization of handovers, we examine the extent to which handover practices of our recorded attending physicians do in fact conform to currently popular structuring schemes that might serve as handover standards. Thus, our research objective is to describe handover communication patterns used by the participating physicians and to compare them to widely promoted, standardized handover communication patterns. Our findings should inform future research, educational and quality improvement activities in the area of handover.

\section{Methods}

\section{Study Design, Setting and Participants}

We conducted a prospective observational study of handover sessions between critical care attending physicians at Kingston General Hospital, a 456-bed tertiary centre in Ontario, Canada, during November 2008 to July 2009 . The ICU is an academic 21 -bed unit providing care for medical, surgical, trauma and cardiovascular surgery patients. In addition to attending physicians, medical teams include residents at different training levels and critical care fellows. Two attending physicians are assigned to the ICU for one-week periods, which start on Fridays. They split patient care and on-call responsibilities during weekdays, while a single physician is in charge during weekends. Attending physician handovers usually take place on Thursdays, typically in the form of oral communication, either in person or, less frequently, over the phone.

We created a data bank by videotaping naturallyoccurring handovers between critical care attending physicians, which usually took place in a conference room within the ICU. In this study, we analyzed a convenience sample of these data consisting of 21 handover sessions. Our study is based on transcriptions of randomly sampled portions of those sessions. Throughout our data collection, we were careful not to alter the behaviour and setting that we were observing. Two of the investigators (RI, DH) participated as subjects in the study by continuing in their role as attending physicians and by conducting handovers as usual. However, all participants were unaware of the research question, as well as the specific nature of the analyses that would be conducted. For each participating physician, one handover session (as an outgoing physician) was randomly selected. From each sampled session, the handovers of four patients were randomly selected using a random number generator. Selected handovers were transcribed; identifiers (e.g., names) were removed from the recordings and transcripts.

This study was approved by the Research Ethics Board of the Queen's University School of Medicine. Signed informed consent was obtained from all participating physicians.

\section{Measures}

Handover activity is conducted in a complex organizational context and can be analyzed at many different levels. Since the commonly promoted handover standards are defined at the patient handover level, our analysis is based on a sampling of patients from within a sample of sessions.

The content of handovers was examined according to three well known standardized communication schemes, including SBAR (Situation, Background, Assessment, Recommendations), SOAP (uujjective, Objective, Assess-

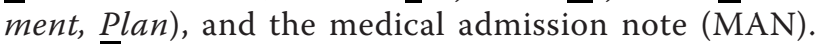
The proportion, absence and recurrence of various elements of the selected standardized schemes were observed and documented.

SBAR is a mnemonic reminding users to present the patient's Situation and Background, followed by the clinician's Assessment and Recommendations. Although SBAR was designed for nurse briefings of physicians in an obstetrics unit that were not handovers [21], the tool has been presumed to be generally useful for the handover situation; it has been widely promoted as a handover standardized procedure [22-26]. In a literature review of 46 papers presenting handover mnemonics, Riesenberg et al found that $69.6 \%$ mentioned SBAR [16]. SOAP is another mnemonic widely espoused by physicians in accordance with the problem-oriented medical record. It reminds physicians to consider and record the patient's Subjective symptoms and complaints, and the Objective physical findings and test results, as well as the physician's Assessment and management Plan [27]. 
SOAP has been suggested as a handover standard [28]. The medical admission note (MAN) regularly used in our hospital (and ICU) includes the following headings: patient identification; chief complaint or reason for ICU admission; history of present illness; past medical history, review of systems and family history; regular medications, habits and allergies; social history; findings on physical examination and test results; and problem formulation and management plan. The MAN, although not specifically designed for handover communication, is likely the most detailed type of medical record and therefore allows for good discrimination between different communication contents.

Questions are generally assumed to have significant potential for improving the quality of handover conversations. For example, questions during handovers may lead to new clinical insights or to error detection and correction $[29,30]$. Some have tried to extend and improve the SBAR scheme by adding a "Q" (for "questions") to the mnemonic [31]. Thus, we coded for the frequency and extent of questions asked by the incoming physicians, treating questions as a distinct element, not overlapping with any of the other standard elements.

There have been many other proposed handover standards, but those we examine here are by far the most common and most likely to match the actual behaviour of our expert participants [1].

The elements composing the various mnemonics were predefined by the research team in accordance with previous publications [24,27] (Figure 1). Transcripts of the selected handovers were then independently color-coded for each of the three communication schemes (Figure 2) by two out of three coders, including a $3^{\text {rd }}$ year medical student, a social worker and a critical care fellow. The coders were trained by the primary investigator (RI) prior to coding the transcripts. The training included in-person instruction regarding the various mnemonics and the definitions of the specific elements, and a

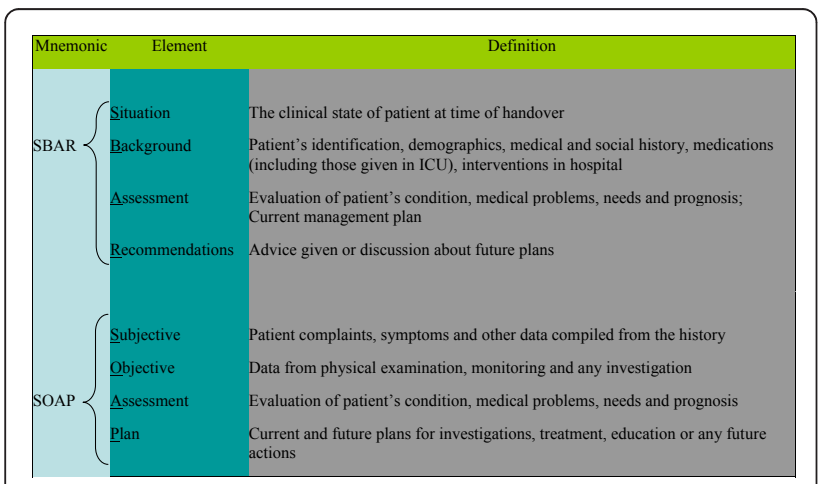

Figure 1 Definitions of SBAR and SOAP.
Out: Had a respiratory arrest upstairs, was intubated upstairs for that (...) despite his poor lungs, he was actually extubated fairly readily (.) about uh (...) 48 hours later and since that time has done okay (.) he has needed bipap (...) on occasion (.) his $\mathrm{C0} 2$ is probably now at a higher set point that it was before (.) his previous baseline I would suspect was in the 60's or 70 's now it seems to be in the 80 's or even as high as 90 (.) with that though, he's alert, completely appropriate and a pH of about, a venous $\mathrm{pH}$ of about

In: Hmokay

Out: So it seems to be his new set point () I suspect he might have had a PE () is the reason his ventilation is now worse () but, because he had been off his Coumadin and now the Coumadin has been restarted we haven't chased the pulmonary embolus down because it's not going to change what we do

In:

Out: $=$ Yes.

In: = where the lower GI bleed came from

Out: Yep, and he hasn't bled since.

Figure 2 Example of a coded transcript according to SBAR and Questions. SBAR, Situation, Background, Assessment,

Recommendation; Out (outgoing), handing over physician; In

(incoming), physician receiving information. Color codes: Green $=$ Situation; Yellow $=$ Background; Light blue $=$ Assessment; Purple $=$ Recommendation; Red = Question; Grey $=$ Other.

supervised coding of one handover transcript. Throughout the coding process, coders' questions and uncertainties were brought to the primary investigator for a final adjudication. There was no attempt to resolve disagreement among the coders. Total duration of handover sessions was measured from the video recording. The quantity of each communication element was measured by word count from the coded transcripts. For the purpose of determining the total word count, all non-alphanumeric content (e.g. punctuation marks) was excluded. Proportions of different elements of the communication schemes were calculated using the number of words or blocks (i.e. consecutive words coded as the same element) relevant to each section over the total number of words or blocks, respectively, in the transcript. Recurrence of specific elements of the various patterns in each patient's handover was defined by counting discontinuous blocks of each element. Demographic data of participating physicians and the setting of each handover were recorded.

\section{Analysis}

Statistical analysis was generated by SAS 9.1.2 (SAS Institute, Cary, North Carolina). Descriptive statistics were used to characterize participant demographic and clinical data, handover characteristics, and proportion and recurrence rates of various elements of the communication schemes. The internal consistency among coders was assessed using the kappa statistic [32]. To 
check for potential differences in approach among physicians, one-way ANOVA was used to examine the variance in handover durations among physicians.

\section{Results \\ Study Sample}

During the study's 34 weeks, a total of 21 handover sessions were recorded, including 15 sessions with a single outgoing and a single incoming physician; 2 sessions with two outgoing and a single incoming; and 4 sessions with two outgoing and two incoming physicians. Ten out of 11 critical care attending physicians agreed to participate in the study and be videotaped. They conducted a mean ( \pm standard deviation) of 2.3 ( \pm 1.3 ) sessions in the outgoing role and were in the incoming role in 2.1 ( \pm 1.4$)$ sessions each. Participating physicians' age, sex, primary specialty and years of experience as an attending physician are shown in Table 1.

\section{General Characteristics}

Nine of the 10 randomly selected handover sessions were performed face to face in a conference room at the ICU; 1 session was performed over the phone. Handover sessions included a mean of $9.5( \pm 2.2)$ patients. Mean durations of the full sessions and of patient-specific handovers were $30 \mathrm{~min} 3 \mathrm{sec}( \pm 11 \mathrm{~min} 7 \mathrm{sec})$ and $2 \mathrm{~min}$ $58 \mathrm{sec}( \pm 57 \mathrm{sec})$, respectively. Handover durations (range, $9 \mathrm{sec}-7 \mathrm{~min} 19 \mathrm{sec}$ ) varied among physicians and $29 \%$ of the variance is explained by the physicians $(p<$ 0.001 ). There was no significant correlation between physicians' years of experience and these handover durations.

Neither the hospital nor the ICU has a policy about how handovers should be conducted. According to their individual preference or practice, outgoing physicians used printed materials, including patient lists and personal notes, while incoming physicians often took notes.

\section{Handover Content}

Table 2 shows the proportion, absence and recurrence rates for SBAR, SOAP and MAN elements, as well as

\begin{tabular}{|c|c|}
\hline Age, mean (SD) & $44.6(8.3)$ \\
\hline Sex, male (\%) & $9(90.0)$ \\
\hline \multicolumn{2}{|l|}{ Primary Specialties, n (\%) } \\
\hline Respirology & $3(30.0)$ \\
\hline Emergency Medicine & $2(20.0)$ \\
\hline General Surgery & $2(20.0)$ \\
\hline Internal Medicine & $2(20.0)$ \\
\hline Anesthesiology & $1(10.0)$ \\
\hline Years as Attending Physician, median (interquartile range) & $9.0(2.5-15.0)$ \\
\hline
\end{tabular}

questions in the handovers. Rates according to two coders and averages of both are presented. Overall level of agreement between the two coders was good for SBAR (Kappa, 0.57), poor for SOAP (Kappa, 0.38) and good for elements of MAN (Kappa, 0.69). In the following lines average rates are reported.

For SBAR, the major part of handover content, 56.4\% ( $\pm 11.6 \%)$, included Background elements. Much of the discordance among coders resulted from disagreement on Situation and Background (Kappa, 0.28). The Recommendation element was completely absent from 22 $(55.0 \%)$ of the patient handovers.

For SOAP, Subjective elements comprised $40.5 \%$ ( \pm $11.4 \%)$ and Assessment 26.0\% ( \pm 8.2\%) of handover content. The Plan element was absent from 3 (7.5\%) of the handovers.

For MAN, expressions of Assessment and descriptions of History of Present Illness comprised 42.9\% ( \pm 7.3\%) and $29.1 \%( \pm 4.3 \%)$, respectively, of handover content. With the exception of Assessment, all MAN elements were occasionally absent from handovers (Table 2).

Questions were asked by the incoming physicians 3.9 $( \pm 3.3)$ times during an average handover and accounted for $5.8 \%( \pm 3.9 \%)$ of the total handover content. Questions were not asked at all in handovers of just 2 (5.0\%) patients.

The proportions, as well as rates of absence and repetitions of all SBAR, SOAP and MAN elements, were similar when excluding data of the two participating investigators (RI, DH) from the analysis.

Table 3 shows the proportions of elements opening ("First") and closing ("Last") the handovers, as well as the arrangement of SBAR, SOAP and MAN elements throughout the handovers, averaged over the two coders.

\section{Opening and Closing}

For SBAR, 77.5\% of the handovers were started with Background and only $13.8 \%$ with Situation information. Only $13.8 \%$ of handovers were concluded with a Recommendation. For SOAP, $66.3 \%$ of the handovers were started with Subjective information and only 26.3\% ended with a Plan. For MAN, the majority (62.5\%) of handovers were started with identifying information, although 20.0\% were started with Assessment and Plan, which also closed most (80.0\%) handovers.

\section{Ordering of Elements}

For SBAR and SOAP, arrangements of the various communication elements were similar: The first 3 elements (SBAR's Situation, Background and Assessment and SOAP's Subjective, Objective and Assessment) followed each other almost randomly. For example, SBAR's Situation was followed by Background information in 38.9\% 
Table 2 Proportion, absence and appearance rates of SBAR, SOAP and MAN elements in patients' handovers

\begin{tabular}{|c|c|c|c|c|c|c|c|c|c|c|}
\hline & & \multicolumn{3}{|c|}{ Proportion, \% ( \pm SD) } & \multicolumn{3}{|c|}{ Absence, $\mathrm{n}(\%)$} & \multicolumn{3}{|c|}{ Appearances per Patient, $n( \pm$ SD) } \\
\hline & & Coder 1 & Coder 2 & Average & Coder 1 & Coder 2 & Average & Coder 1 & Coder 2 & Average \\
\hline & $S$ & $5.2(5.6)$ & $26.3(14.4)$ & $15.8(8.4)$ & $8(20.0)$ & $1(2.5)$ & $1(2.5)$ & $2.1(1.8)$ & $4.5(2.8)$ & $3.3(2.1)$ \\
\hline \multirow[t]{4}{*}{ SBAR } & B & $67.6(11.2)$ & $45.3(15.1)$ & $56.4(11.6)$ & $0(0.0)$ & $0(0.0)$ & $0(0.0)$ & $4.9(2.6)$ & $3.5(2.2)$ & $4.2(2.2)$ \\
\hline & A & $18.8(8.9)$ & $20.3(10.1)$ & $19.5(9.0)$ & $1(2.5)$ & $2(5.0)$ & $1(2.5)$ & $4.3(2.6)$ & $4.5(2.6)$ & $4.4(2.2)$ \\
\hline & $\mathrm{R}$ & $2.6(3.9)$ & $2.3(3.8)$ & $2.4(3.7)$ & $23(57.5)$ & $25(62.5)$ & $22(55.0)$ & $0.5(0.7)$ & $0.4(0.6)$ & $0.5(0.6)$ \\
\hline & $\mathrm{S}$ & $26.2(11.1)$ & $54.4(17.3)$ & $40.5(11.4)$ & $1(2.5)$ & $1(2.5)$ & $1(2.5)$ & $2.9(1.6)$ & $7.4(4.1)$ & $5.1(2.5)$ \\
\hline \multirow[t]{7}{*}{ SOAP } & $\mathrm{O}$ & $18.2(9.7)$ & $13.6(9.3)$ & $15.9(7.2)$ & $2(5.0)$ & $1(2.5)$ & $0(0.0)$ & $4.1(2.7)$ & $4.7(3.1)$ & $4.4(2.7)$ \\
\hline & A & $31.8(14.1)$ & $20.6(9.2)$ & $26.0(8.2)$ & $0(0.0)$ & $1(2.5)$ & $0(0.0)$ & $5.0(3.3)$ & $5.3(3.5)$ & $5.2(3.0)$ \\
\hline & P & $17.7(11.3)$ & $5.6(6.5)$ & $11.6(8.0)$ & $3(7.5)$ & $17(42.5)$ & $3(7.5)$ & $2.8(2.0)$ & $1.2(1.5)$ & $2.0(1.4)$ \\
\hline & ID & $1.8(0.9)$ & $2.1(0.7)$ & $2.0(0.7)$ & $8(20.0)$ & $6(15.0)$ & $6(15.0)$ & $0.9(0.5)$ & $1.2(0.7)$ & $1.0(0.6)$ \\
\hline & $\mathrm{CC}$ & $0.6(0.5)$ & $0.3(0.4)$ & $0.5(0.4)$ & 33 (82.5) & $36(90.0)$ & $35(87.5)$ & $0.2(0.4)$ & $0.1(0.3)$ & $0.1(0.3)$ \\
\hline & $\mathrm{HPI}$ & $24.6(6.9)$ & $33.5(4.0)$ & $29.1(4.3)$ & $1(2.5)$ & $1(2.5)$ & $1(2.5)$ & $3.4(2.1)$ & $9.2(4.8)$ & $6.3(4.7)$ \\
\hline & $\mathrm{PMH}$ & $5.7(3.7)$ & $2.0(1.7)$ & $3.8(2.4)$ & $14(35.0)$ & $25(62.5)$ & $20(50.0)$ & $1.3(1.4)$ & $0.8(1.2)$ & $1.0(1.4)$ \\
\hline \multirow[t]{6}{*}{ MAN } & $\mathrm{MHA}$ & $2.2(1.6)$ & $0.5(0.7)$ & $1.3(1.0)$ & $20(50.0)$ & $33(82.5)$ & $27(67.5)$ & $0.9(1.3)$ & $0.3(0.8)$ & $0.6(1.1)$ \\
\hline & Social & $2.0(2.0)$ & $3.1(3.5)$ & $2.5(2.5)$ & $28(70.0)$ & $21(52.5)$ & $25(62.5)$ & $0.5(0.8)$ & $0.9(1.2)$ & $0.7(1.1)$ \\
\hline & $\mathrm{DOC}$ & $3.7(4.5)$ & $0.8(0.8)$ & $2.2(2.5)$ & $29(72.5)$ & $33(82.5)$ & $31(77.5)$ & $0.5(1.0)$ & $0.3(0.6)$ & $0.4(0.8)$ \\
\hline & $P E$ & $3.5(1.7)$ & $4.5(2.8)$ & $4.0(1.9)$ & $16(40.0)$ & $11(27.5)$ & $14(35.0)$ & $1.0(1.1)$ & $1.9(2.1)$ & $1.5(1.7)$ \\
\hline & Tests & $12.6(6.6)$ & $10.5(4.9)$ & $11.6(5.5)$ & $8(20.0)$ & $6(15.0)$ & 7 (17.5) & $2.9(2.6)$ & $3.3(2.8)$ & $3.1(2.7)$ \\
\hline & $A+P$ & $43.2(9.4)$ & 42.7 (6.7) & $42.9(7.3)$ & $0(0.0)$ & $0(0.0)$ & $0(0.0)$ & $7.4(4.1)$ & $11.4(6.5)$ & $9.4(5.8)$ \\
\hline \multicolumn{2}{|c|}{ Questions } & $5.7(3.9)$ & $5.9(3.9)$ & $5.8(3.9)$ & $2(5.0)$ & $2(5.0)$ & $2(5.0)$ & 3.9 (3.6) & $3.9(3.3)$ & 3.9 (3.3) \\
\hline
\end{tabular}

SBAR, Situation, Background, Assessment, Plan; SOAP, Subjective, Objective, Assessment, Plan; MAN, medical admission note; SD, standard deviation; ID, identification; CC, chief complaint; HPI, history of present illness; $\mathrm{PMH}$, past medical history; MHA, medications, habits and allergies; DOC, directives of care; PE, physical examination; $\mathrm{A}+\mathrm{P}$, assessment and plan.

and by Assessment in $57.4 \%$ of the handovers. The last elements (SBAR's Recommendation and SOAP's Plan) followed any of the other elements to a lesser extent. For MAN, most elements were followed by Assessment and Plan. Exceptions include Identification, which was usually followed by History of Present Illness (51.4\%) or Past Medical History (32.4\%); Chief Complaint, which was absent in $87.5 \%$ of the handovers; and Past Medical History, which was usually followed by History of Present Illness $(58.1 \%)$. Only a single element, Tests, was followed by the "appropriate" element (in this case Assessment and Plan, which, in fact, followed most other elements) in most handovers (56.3\%).

Figure 3 and Figure 4 show the occurrence of SBAR and SOAP elements, respectively, in the 40 handovers that we analyzed. In addition to the dominance of Background and Subjective elements (for SBAR and SOAP, respectively), these figures illustrate the mixed arrangement and multiple occurrences of all the mnemonic elements.

Figure 5 and Figure 6 represent the proportion of SBAR, SOAP and Question elements in each ten percent block of handover length averaged over 40 handovers and 2 coders. Figure 5 shows that Background information comprises about $90 \%$ of the content in the first 20-30 percent of an average handover, and then gradually decreases throughout the handover. Situation content is present in a fairly constant level whereas expressions of Assessment gradually increase throughout the handovers. Recommendations first appear at the second half of an average handover and then gradually increase. Figure 6 shows that Subjective aspects of the patient are the vast majority of material in the first 10 percent of an average handover, but fall to about 30 percent of the content by the middle of the handover and, on average, remain at about that level for the rest of the discussion. Objective elements, in contrast, are present at a moderate but constant level throughout the 40 handovers.

\section{Discussion}

During end-of-week handovers in the ICU, the observed attending physicians spent about 3 minutes discussing each patient. Elements of the three communication schemes (SBAR, SOAP, MAN), often completely absent, were distributed throughout the handovers.

Although SBAR, originally developed to achieve effective briefing during critical situations [21,24], is relatively new to the medical community, SOAP and MAN have been commonly promoted and could potentially be the physicians' default for the purpose of handover communication. Clearly, these physicians were taking a communication approach that is not standardized around a simple ordered list of topics. Apparently, these widely 
Table 3 Proportion of elements opening ("First") and closing ("Last") the handovers and succession of SBAR, SOAP and MAN elements*, @

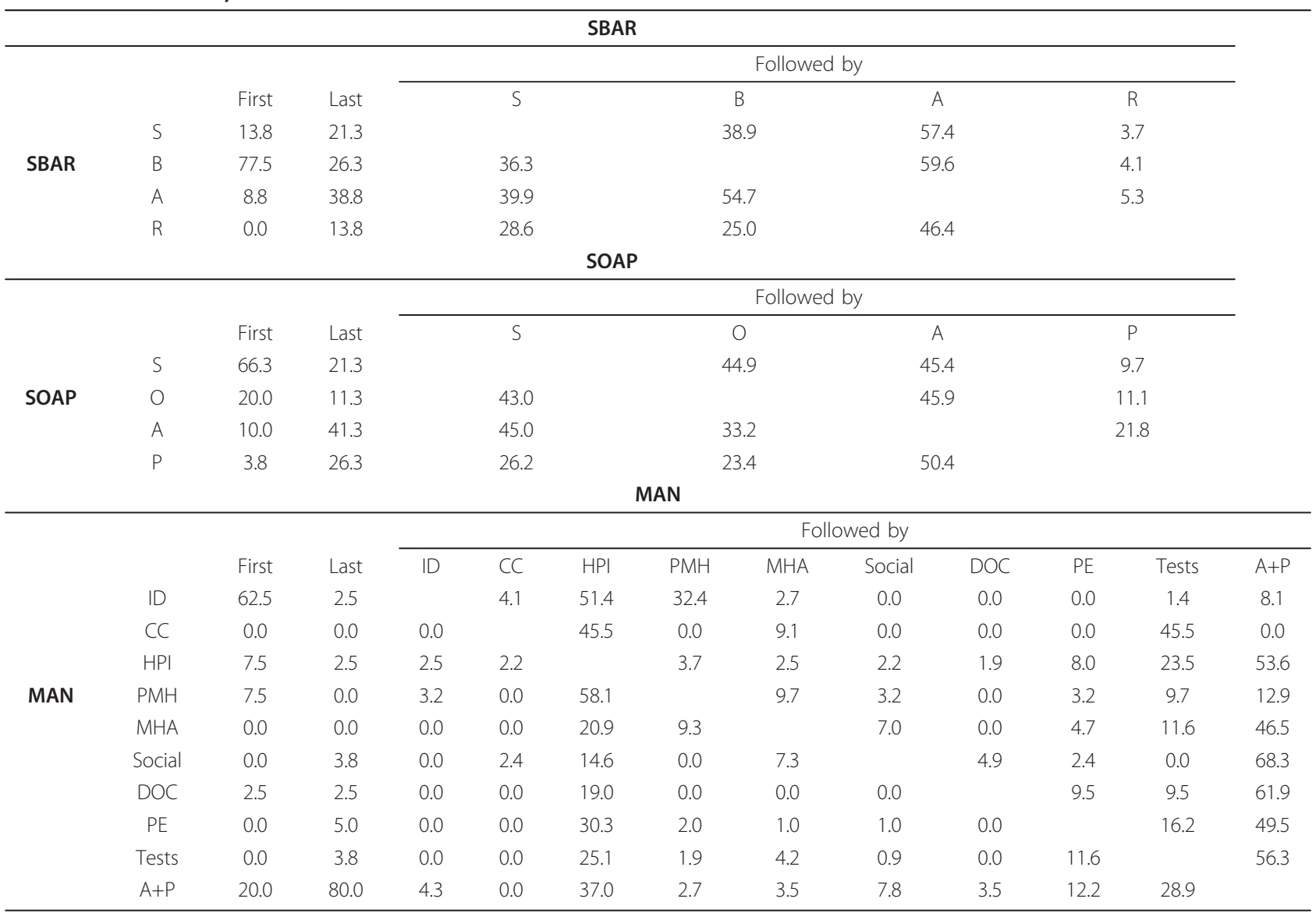

* Averaged over the two coders; all numbers are in percentages. SBAR, Situation, Background, Assessment, Plan; SOAP, Subjective, Objective, Assessment, Plan; MAN, medical admission note; ID, identification; CC, chief complaint; HPI, history of present illness; PMH, past medical history; MHA, medications, habits and allergies; Social, social history; DOC, directives of care; $\mathrm{PE}$, physical examination; $\mathrm{A}+\mathrm{P}$, assessment and plan.

${ }^{\circledR}$ An example for data interpretation: For SBAR, Background elements opened $77.5 \%$ and closed $26.3 \%$ of the handovers. Whenever Background elements appeared and were not last, they were followed by Situation, Assessment, and Recommendation elements in $36.3 \%$, 59.6\%, and $4.1 \%$ of the opportunities, respectively.

promoted schemes do not fit the clinical work required during handover.

Information about the patient's condition and history (Situation and Background elements in SBAR; Subjective and Objective in SOAP; and History of Present Illness, Test Results and Past Medical History elements in the MAN) comprised about half of the handovers' contents. Considering a usual week-long trajectory of critically ill patients in the ICU, the high proportion of "background" information in the handovers is not surprising. Interpretation of the patient's condition (Assessment in SBAR, SOAP and MAN) comprised about one third of the discussion.

Questions were asked commonly by incoming physicians: about 4 questions during each handover; questions not asked at all in only 2 out of the 40 handovers. Although the value of questions is often assumed to lie in verifying or completing the transmission of facts about the patient, questions may play a considerably larger role in the process of two physicians jointly constructing their picture of the patient. Questions can invite or solicit more information; initiate repair or correction of mistakes; seek confirmation of hearing and/or understanding; slow the conversation down for the purpose of note-taking; propose an alternative organization of information; display expertise or assert competence (someone knows enough to ask the question); provide a polite way of suggesting an alternative diagnosis or treatment possibility; and so forth. Through questions and other responses, incoming physicians become the co-authors of reports by outgoing physicians. Handovers are an interactive accomplishment, not a monologic and unilateral transfer of information. Furthermore, what constitutes a question is not always clear: human speech does not come with explicit question marks, so the occurrence of a question may be ambiguous, and that 


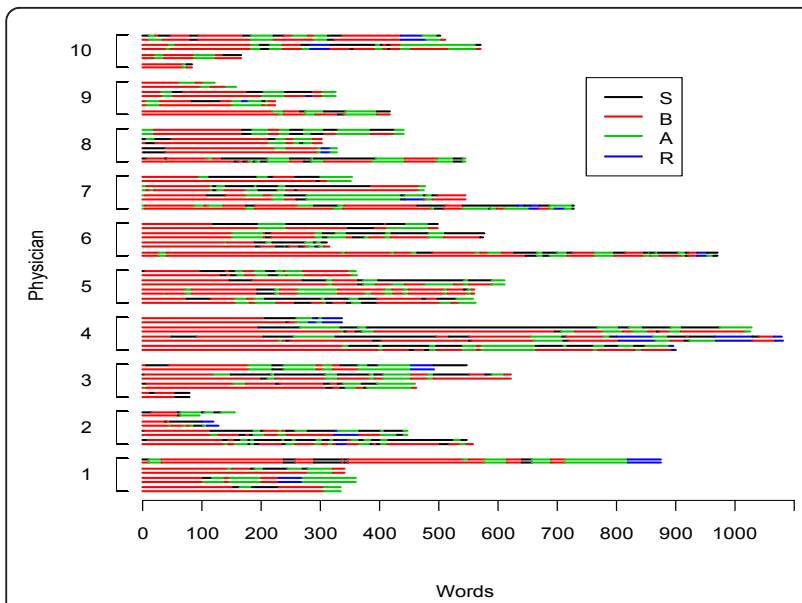

Figure 3 Occurrence of SBAR elements. Two coders shown for four sampled patient handovers for each of 10 handing-over physicians.* S, Situation; B, Background; A, Assessment; R, Recommendation. ${ }^{*}$ Data are for the conversation in which physician (i) was handing over, but the data include what was said in the conversation by the incoming physician as well.

ambiguity may serve the micro-political ends of the participants, allowing for such things as diplomatic corrections or soft directions. Further research is needed to refine and analyze the types and functions of questions in handovers.

In our recorded handovers, different elements of the various communication schemes were continuously interwoven throughout the exchanges. This may suggest that the physicians chose to deliver their message by portraying a sensemaking, relevant story rather than

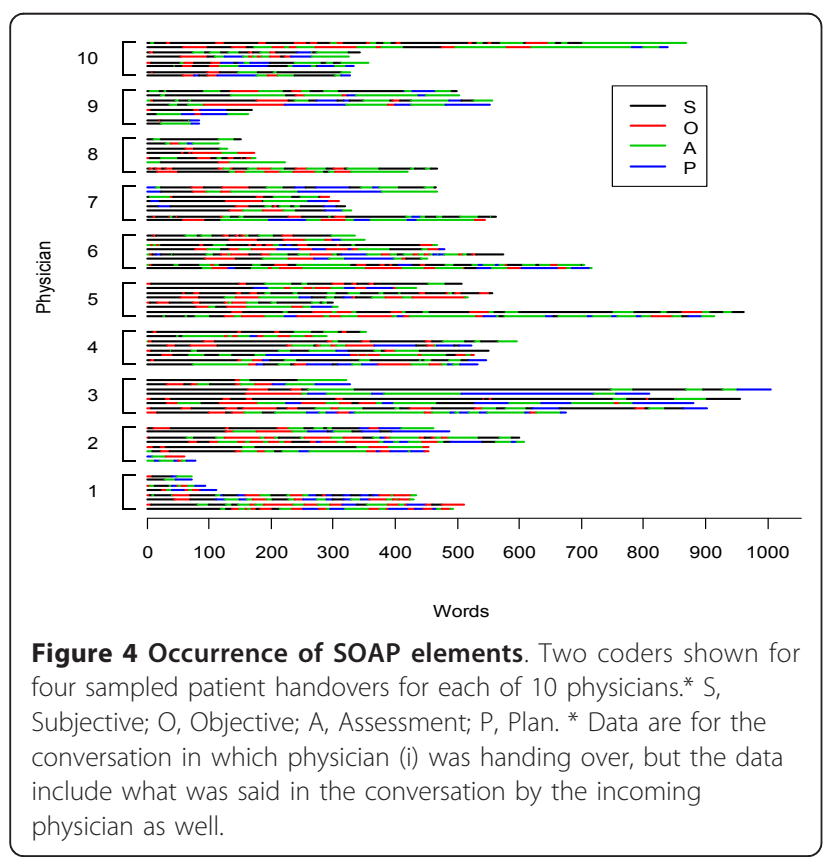

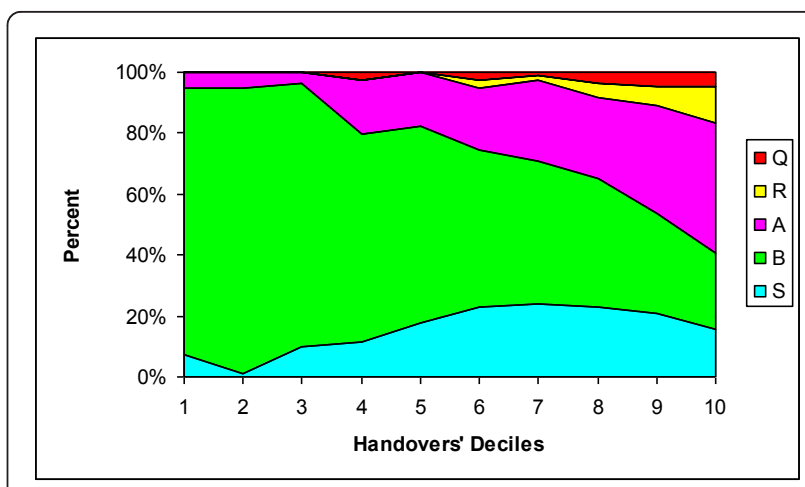

Figure 5 Proportion of $S B A R+Q$ elements in each ten percent block of standardized handover length, averaged over $\mathbf{4 0}$ handovers and 2 coders. S, Situation; B, Background; A, Assessment; R, Recommendation; Q, Question.

spelling out the entirety of the available information about their patients $[29,33]$. An account of events leading to the patient's current condition is likely to include multiple segments of various types of information, as suited to the individual patient's medical problems. Utterances of medical history, test results and their interpretation, social history, directives of care, etc. may need to be carefully deployed-placed in narrative context, omitted in some cases, repeated in others-to achieve the supposed goal of a handover: an understanding of the patient's condition and needs, by the incoming physician, in a way that is memorable and can usefully inform the subsequent actions [3,34]. Attempting to rigidly apply any of the studied standardized approaches for delivering a handover might have failed to achieve this goal.

One of the most striking findings in our study relates to elements that were absent in handovers. In particular, Recommendation content was absent in about $60 \%$ of patient handovers, in the judgement of both coders.

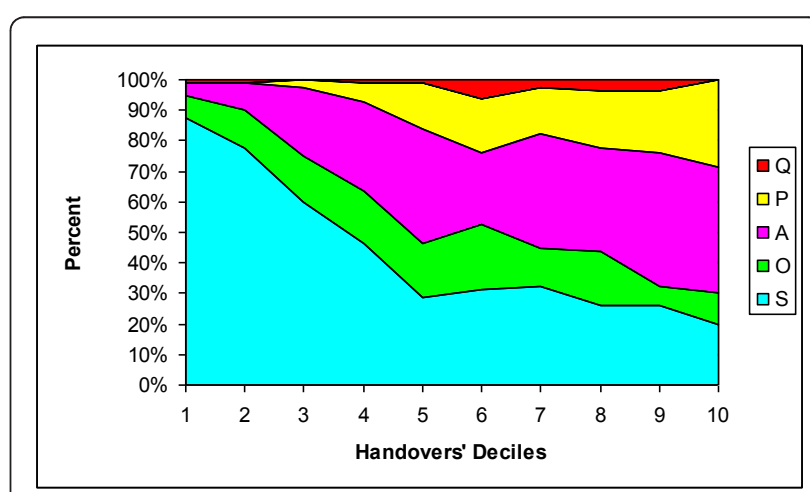

Figure 6 Proportion of SOAP+Q elements in each ten percent block of standardized handover length, averaged over $\mathbf{4 0}$ handovers and 2 coders. S, Subjective; O, Objective; A, Assessment; P, Plan; Q, Question. 
Lack of recommendation has also been observed in nurses practicing SBAR [35]. There are several possible explanations for this finding. First, it could be that the outgoing physicians have inadvertently omitted recommendations (i.e. error of omission) [36,37]. Second, because advice-giving and correcting may undermine the recipient's status [38], it may be that outgoing physicians did not offer recommendations out of respect for the incoming physicians' professional status $[39,40]$. Third, it may be that the outgoing physicians focused on conveying their sense of the patient's trajectory of care and what should be done next (recommendations) in narrative or story form, rather than a detailed list of what is wrong with the patient and what to do next $[33,41,42]$. Fourth, it may be that outgoing physicians sought to draw attention to potential problems of the patient even if they didn't have a clear sense of how to resolve those problems. Regardless of the reasons, a complete lack of recommendations or mention of management plans might lead to misperception by the incoming physician, and possibly to undesirable outcomes for patients and the organization. Further research is warranted into the reasons for and consequences of lack of peer-to-peer dialogue on treatment recommendations and into strategies that might improve physicians' discussion of such content.

For various reasons the quality of these recorded handovers cannot be evaluated at present. First, due to the complexity of the communication, and to the absence of the appropriate research, a "gold standard" for handover communication does not exist. Second, our small sample does not allow for a meaningful evaluation of clinical outcomes such as physician satisfaction, errors, indicators of inefficiency and adverse events. Further research is required to identify quality indicators for handovers. However, we believe that the observed communication of the studied physicians does indicate important features of expertise. It is unlikely that content elements were interspersed throughout the handovers simply because these attending physicianswith an average of 9 years of experience-lacked the ability to organize their thoughts and put together a coherent description of their patients. In fact, within about 3 minutes they summarized several days in the history of a critically ill patient. We cautiously suggest that the apparent "disorganization" could be reflecting a more sophisticated approach to handovers: a logical, efficient communication of complex information. Further research is required to examine whether alternative approaches to handover communication can be identified among the studied physicians and to identify features associated with good handovers. If such approaches and features can be identified, the implications for medical schools, training programs and practicing physicians would be substantial.

This study has several important strengths. First, to our knowledge, this is the only systematic description of medical handovers as performed by full-time faculty attending physicians. Experience is expected to engender wisdom and expertise, which, in turn, would contribute to the quality of handovers. Better understanding of high quality handovers will inform educational and quality improvement efforts in the area. Second, our description is one of very few (e.g. $[7,43])$ based on video or audio recordings of naturally occurring handovers (i.e., handovers that would have occurred whether or not the recorder was present).

Our study has four known limitations. First, participating physicians were aware of being recorded and this, similar to the Hawthorne Effect, might have influenced their behaviour. In addition, the set of sessions captured in the archive, while quite extensive, was nonetheless a convenience sample and thus not all handovers were captured during the study period. Furthermore, two of the participating physicians were also investigators in the study. These factors may have detracted from the internal validity of our findings. However, the studied handovers were recorded with the initial objective to create a data bank of naturally occurring handovers. Specific research questions did not exist and were not discussed prior to the completion of the data bank. It is likely that the recorded physicians attempted to do their best at the handovers; however, in the absence of any predefined outcome measures, participants could not have been biased in any way with respect to our research question. A second limitation is that the study was performed in a single centre and included a relatively small number of physicians. Our findings may, therefore, have limited generalizability to other ICUs. However, there is no institutional protocol around the format of handovers at Kingston General Hospital and all ten critical care physicians we studied have worked in other hospitals, which suggests that their behavior is likely more broadly representative. Third, there was substantial disagreement between coders regarding some elements of the standardized communication schemes. Although this may reflect the complexity of the handed over information and the inherent ambiguity of the proposed standard categories, it is nonetheless clear that standardized schemes were not used by the studied physicians according to any of the coders. All coders agreed that blocks of each content type were widely scattered throughout each patient discussion. We should perhaps not be surprised that coders have some difficulty agreeing as medical interns being taught such schemes also have difficulty following them [11]. In addition, there 
are some noticeable discrepancies among the coding according to the different communication standards. For example, whereas SBAR's Recommendation was absent in $55 \%$ of the handovers, SOAP's Plan, a seemingly equivalent construct, was absent in only $7.5 \%$. Similarly, the combined proportion of SBAR's Assessment and Recommendation (21.9\%) is much smaller, according to both coders, than the apparently similar content represented by SOAP's Assessment and Plan (37.6\%). These can be explained by differences in the definitions of similar elements of the different standards (Figure 1). Finally, the quality of the recorded handovers was not assessed and consequently a relationship between handover quality and clinical outcomes could not be examined. Since a validated measurement tool for handover quality does not exist, further research is required to identify quality indicators for handover communication.

\section{Conclusion}

Faculty-level critical care physicians did not follow commonly recommended medical communication schemes (SBAR, SOAP, MAN) during naturally occurring, videotaped handovers. Statements corresponding to the various mnemonic elements were scattered throughout the handovers without block structure or fixed order of arrangement and were sometimes entirely absent. Evidently, these widely promoted schemes do not fit the clinical work of handover within the present setting. Further research is required to examine whether a typical communication approach can be identified in the recorded handovers; to explore the role of questions and active involvement of incoming physicians; to evaluate the handovers' quality and relevance to meaningful clinical outcomes; and to identify features of high-quality handover communication.

\section{List of abbreviations}

A+P: assessment and plan; CC: chief complaint; DOC: directives of care; ICU: intensive care unit; ID: identification; HPI: history of present illness; MAN: medical admission note; MHA: medications: habits and allergies; PE: physical examination; PMH: past medical history; SBAR: situation: background: assessment: recommendations; SD: standard deviation; SOAP: subjective, objective, assessment, plan.

\section{Acknowledgements}

This project received funding from the Physicians' Services Incorporated Foundation

\footnotetext{
Author details

'Department of Medicine and Critical Care Program, Queen's University, Kingston General Hospital, Etherington Hall, Room 1005, 94 Stuart Street, Kingston, ON, Canada, K7L 3N6. ${ }^{2}$ Department of Organizational Leadership \& Strategy, Marriott School of Management, Tanner Building 790, Brigham Young University, Provo, Utah 84602, USA. 'Rotman School of Management, 105 St. George Street, Toronto, ON, Canada, M5S 3E6. ${ }^{4}$ Clinical Research Centre, Kingston General Hospital, Kingston, ON, Canada, K7L 3N6. ${ }^{5}$ School of Information, 312 West Hall, School of Public Policy, 407 Lorch Hall, University of Michigan, Ann Arbor, Michigan 48109-1092, USA.
}

\section{Authors' contributions}

$\mathrm{Rl}, \mathrm{CLB}, \mathrm{MC}, \mathrm{DH}$ and $\mathrm{MC}$ conceived the study, participated in data analysis and interpretation and drafted the manuscript. RI also supervised data collection. AD helped with statistical analysis and interpretation.

All authors read and approved the final manuscript.

\section{Competing interests}

The authors declare that they have no competing interests.

Received: 11 May 2011 Accepted: 10 January 2012

Published: 10 January 2012

\section{References}

1. Cohen MD, Hilligoss PB: Handoffs in hospitals: A review of the literature on information exchange while transferring patient responsibility or control. 2009 [http://hdl.handle.net/2027.42/61522].

2. Sutcliffe KM, Lewton E, Rosenthal MM: Communication failures: An insidious contributor to medical mishaps. Acad Med 2004, 79(2):186-94.

3. Arora V, Johnson J, Lovinger D, Humphrey HJ, Meltzer DO: Communication failures in patient sign-out and suggestions for improvement: A critical incident analysis. Qual Saf Health Care 2005, 14(6):401-7.

4. Gandhi TK: Fumbled handoffs: One dropped ball after another. Annals of Internal Medicine 2005, 142(5):352-8.

5. Shojania KG, Fletcher KE, Saint S: Graduate medical education and patient safety: A Busy-and occasionally Hazardous-Intersection. Annals of Internal Medicine 2006, 145(8):592-8.

6. Singh $H$, Thomas EJ, Petersen LA, Studdert DM: Medical errors involving trainees: A study of closed malpractice claims from 5 insurers. Arch Intern Med 2007, 167(19):2030-6.

7. Horwitz LI, Moin T, Krumholz H, Wang L, Bradley EH: Consequences of inadequate sign-out for patient care. Arch Intern Med 2008, 168(16):1755-60.

8. Safe handover: Safe patients. London: British Medical Association; 2004 [http://www.bma.org.uk/images/safehandover_tcm41-20983.pdf].

9. Solet DJ, Norvell JM, Rutan GH, Frankel RM: Lost in translation: Challenges and opportunities in physician-to-physician communication during patient handoffs. Acad Med 2005, 80(12):1094-9.

10. Vidyarthi AR, Arora V, Schnipper JL, Wall SD, Wachter RM: Managing discontinuity in academic medical centers: Strategies for a safe and effective resident sign-out. J Hosp Med 2006, 1(4):257-66.

11. Horwitz LI, Moin T, Green ML: Development and implementation of an oral sign-out skills curriculum. J Gen Intern Med 2007, 22(10):1470-4.

12. Arora V, Manjarrez E, Dressler D, Basaviah P, Halasyamani L, Kripalani S: Developing hand off standards for hospitalists. Society of Hospital Medicine 2007.

13. Arora VM, Farnan JM: Care transitions for hospitalized patients. Med Clin North Am 2008, 92(2):315-24.

14. 2008 national patient safety goals hospital program. 2008 [http://www jointcommission.org/standards_information/npsgs.aspx].

15. Action on patient safety: High 5s. World Health Organization; [http://www. who.int/patientsafety/solutions/high5s/High5_overview.pdf].

16. Riesenberg LA, Leitzsch J, Little BW: Systematic review of handoff mnemonics literature. Am J Med Qual 2009, 24(3):196-204.

17. Riesenberg LA, Leitzsch J, Massucci JL, Jaeger J, Rosenfeld JC, Patow C, et al: Residents' and attending physicians' handoffs: A systematic review of the literature. Acad Med 2009, 84(12):1775-87.

18. de Certeau M: The Practice of Everyday Life. Berkeley, CA: University of California Press; 1984

19. Orbuch TL: People's accounts count: The sociology of accounts. Annu Rev Sociol 1997, , 23: 455-78

20. Orlikowski WJ: Knowing in practice: Enacting a collective capability in distributed organizing. Organization Science 2002, 13(3):249-73.

21. Profiles in improvement: Doug bonacum of kaiser permanente. Institute for Healthcare Improvement; 2008 [http://www.hi.org/knowledge/Pages/ AudioandVideo/ProfilesinImprovementDougBonacumofKaiserPermanente. aspx].

22. Wakefield DS, Cyphert ST, Murray JF, Uden-Holman T, Hendryx MS, Wakefield BJ, et al: Understanding patient-centered care in the context of total quality management and continuous quality improvement. $J t$ Comm J Qual Improv 1994, 20(3):152-61. 
23. Feldman JA: Medical errors and emergency medicine: Will the difficult questions be asked, and answered? Acad Emerg Med 2003, 10(8):910-1.

24. SBAR technique for communication: A situational briefing model. 2006 [http://www.ihi.org//HI/Topics/PatientSafety/SafetyGeneral/Tools/ SBARTechniqueforCommunicationASituationalBriefingModel.htm].

25. Dunn W, Murphy JG: The patient handoff: Medicine's formula one moment. Chest 2008, 134(1):9-12.

26. White RS, Hall DM: The Bermuda triangle healthcare. An Illinois healthcare system closes the gaps in patient handoff communication. Health Manag Technol 2008, 29(7):30-1.

27. Weed LL: Medical records, medical education, and patient care: The problem-oriented medical record as a basic tool. Cleveland, $\mathrm{OH}$ : Case Western Reserve University; 1970.

28. Kilpack V, Dobson-Brassard S: Intershift report: Oral communication using the nursing process. J Neurosci Nurs 1987, 19(5):266-70.

29. Christianson MK: Updating as part of everyday work: An interactional perspective. 2009 [http://hdl.handle.net/2027.42/64680].

30. Wears RL, Perry SJ, Shapiro M, Beach C, Behara R: Shift changes among emergency physicians: Best of times, worst of times. Human Factors and Ergonomics Society, 47th Annual Meeting Denver, CO.; 2003.

31. Runy LA: Patient handoffs. Hosp Health Netw 2008, 82(5):40-7.

32. Fleiss JL, Levin B, Paik MC: Statistical methods for rates and proportions (3rd ed.). New York: John Wiley \& Sons; 2003.

33. Weick KE, Sutcliffe KM, Obstfeld D: Organizing and the process of sensemaking. Organization Science 2005, 16(4):409-21.

34. Borowitz SM, Waggoner-Fountain LA, Bass EJ, Sledd RM: Adequacy of information transferred at resident sign-out (in-hospital handover of care): A prospective survey. Qual Saf Health Care 2008, 17(1):6-10.

35. Landro A: Hospitals combat errors at the 'hand-off'. WSJ 2010 [http:// online.wsj.com/article/SB115145533775992541.html], 2006:September 8.

36. Horwitz LI, Moin T, Krumholz HM, Wang L, Bradley EH: What are covering doctors told about their patients? Analysis of sign-out among internal medicine house staff. Qual Saf Health Care 2009, 18(4):248-55.

37. Miller A, Scheinkestel C, Limpus A, Joseph M, Karnik A, Venkatesh B: Uniand interdisciplinary effects on round and handover content in intensive care units. Hum Factors 2009, 51(3):339-53.

38. Ende J, Pomerantz A, Erickson F: Preceptors' strategies for correcting residents in an ambulatory care medicine setting: A qualitative analysis. Acad Med 1995, 70(3):224-9.

39. Cicourel AV: Cognitive and organizational aspects of medical diagnostic reasoning. Discourse Processes 1987, 10(4):347-67.

40. Abbott A: The order of professionalization. Work Occup 1991, 18(4):355-84

41. Browning LD: Lists and stories as organizational communication. Communication Theory 1992, 2(4):281-302.

42. Weick KE: Sensemaking in organizations. Thousand Oaks, CA: Sage Publications; 1995.

43. Kerosuo $\mathrm{H}$ : Boundaries in action: An activity-theoretical study of development, learning, and change in health care organization for patients with multiple and chronic illnesses. Helsinki: University of Helsinki, Faculty of Behavioural Sciences, Department of Education, Center for Activity Theory and Developmental Work Research; 2006 [https://www. doria.fi/handle/10024/3666

Pre-publication history

The pre-publication history for this paper can be accessed here: http://www.biomedcentral.com/1472-6963/12/11/prepub

doi:10.1186/1472-6963-12-11

Cite this article as: Ilan et al.: Handover patterns: an observational study of critical care physicians. BMC Health Services Research 2012 12:11.

\section{Submit your next manuscript to BioMed Central and take full advantage of:}

- Convenient online submission

- Thorough peer review

- No space constraints or color figure charges

- Immediate publication on acceptance

- Inclusion in PubMed, CAS, Scopus and Google Scholar

- Research which is freely available for redistribution

Submit your manuscript at www.biomedcentral.com/submit 